\title{
Systematic slope stability assessment through deformation field monitoring
}

\author{
Yukun $\mathrm{Wei}^{1}$, Anders B. Lundberg ${ }^{2 *}$, and Fredrik Resare ${ }^{2}$ \\ ${ }^{1}$ Golder Associates AB, PO Box 20127, 10460 Stockholm, Sweden \\ ${ }^{2}$ ELU Konsult AB, PO Box 27006, 102 51, Stockholm, Sweden
}

\begin{abstract}
Field monitoring is frequently carried out during excavations and other geotechnical activities and provides additional information during the execution of a construction project. The interpretation of field monitoring data is often obscured by measurement noise and disturbance, and a systematic approach to assess both the quality and implications of the field monitoring data is very helpful in geotechnical practice. The possibility to infer practical conclusions from the field monitoring data depends on the type of field measurements, especially in monitoring of the stability of slopes. Pore pressure measurements can serve as a direct measurement of utilized soil strength for a slope, while deformation measurements are significantly more ambiguous and complicates the interpretation. The assessment of slope stability through field monitoring of deformations requires inverse or back analysis of the soil properties, followed by a forward analysis of the resulting slope stability. Such an inverse or back analysis is frequently influenced by nonuniqueness of the material properties and the stability of the measurement data. Systematic approaches to inverse or back analysis have been demonstrated in the scientific literature, but the practical use of these methods is not entirely straight-forward. The current paper presents a case study of systematic slope stability assessment through field measurements of deformations with a review of the field monitoring programme, numerical simulations of deformations, and a simplified approach to back analysis of the soil parameters. The excavation of a slope in an urban environment including layers of organic clay covered with highly heterogeneous gravel fill is used as an example of geotechnical back analysis. The aim is to elucidate some of the challenges in geotechnical back analysis while providing some practical solutions for practice.
\end{abstract}

\section{Introduction}

Field measurements and observations have long formed a significant part of geotechnical engineering, [1]. The development of sophisticated computational methods has made inverse or back-analysis of geotechnical observations available in geotechnical engineering practice, e.g. [2]-[5]. Slope stability analysis constitutes a common geotechnical problem, and observations of slope have been performed on many locations, resulting in added information about the behaviour of the slope [6]-[8].

The use of back-analysis for slope stability however requires advanced computational methods because of the non-linear response of soils at the onset of slope collapse when the soil mass reaches plastic yield, [9]. Slope collapses are frequently governed by quickly developing highly localized deformation zones involving a strength degradation of the soil, making detailed back-analysis between deformations and soil parameters cumbersome, [10-11]. Detailed back-analysis in such cases is prohibited by instability and non-uniqueness of the numerical solutions, as well as multicollinearity of the input parameters. The instability originates from the non- linear response of the mechanical system, in which the solutions are highly influenced by the input parameters. More detailed constitutive models include a wide range of parameters, which results in non-uniqueness of the back-analysis and in which several sets of parameters give a decent back-analysis, but result in very different forward predictions, [4]. Multicollinearity is the result of high intercorrelations in the explanatory variables, and influences parameter estimation and forward prediction, [12].

Assessing the validity of parameter estimation and forward prediction depends on the epistemological perspective, [13-14]. Back-analysis can result in reasonable predictions, but the justification of the specific prediction depends on the belief of the prediction, [15]. Since design codes require slopes to be very unlikely to collapse, experimental verification of the validity of the prediction is in most cases not possible. Calculation models are validated through simulations of real slope collapses, but uncertainties in the input data is often significant, e.g. [10]. The justified belief of the parameter estimation and the forward prediction of the back-analysis consequently depends on the specific field conditions and the calculation

\footnotetext{
* Corresponding author: anders.beijer@elu.se
} 
methodology, and the field conditions where the specific calculation model was validated. The uncertainties are therefore considerable, and justified belief in the resulting forward prediction is consequently often not attainable more than a subjective statement due to contradiction in the input-data and field measurements, [15-16].

The aim of the current contribution is to elucidate some of the uncertainties involved in back-analysis of slope stability. A case study of a slope equipped with field monitoring equipment is presented, and a simplified semi-probabilistic parameter estimation and forward prediction framework is presented. The large model input uncertainties in the slope stability analysis framework is discussed in detail, and some practical methods to alleviate this factor in the modelling phase are elaborated.

\section{Geotechnical back-analysis}

Inverse or back-analysis encompasses the estimation of model parameters with the model output as input [2-3]. Properties estimated from inverse analysis can be used as subsequent input in forward predictions with the same calculation model, shown schematically in Figure 1. Inverse analysis hence calibrates a numerical model with respect to the field measurement so that the uncertainties in the model can be reduced, [4].

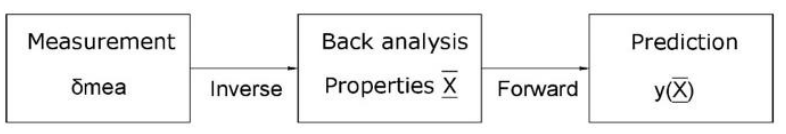

Fig. 1. Inverse analysis of model parameters and subsequent forward prediction.

Inverse analysis methods are here classified as direct or indirect models, depending to the relationship between input model parameters and field data. Direct models are available for linear relationships between modelled parameters and field measurements with a oneon-one transformation is possible, [2]. In most cases, observations display some scatter but linear minimization technique such as the least-squares method are available, [3]. Indirect methods are applied for nonlinear and non-unique relationships between modelled parameters and data, [17]. If a nonlinear plastic relationship exists, the model input and output are not proportional and model interpretation is hampered. Therefore, an alternative way is developed by minimizing the absolute difference between measured data and calculated data to find estimated values for the studied parameters [5]. In the following section a casestudy using a simplified indirect inverse method will be demonstrated.

\section{Case study}

A case study concerning slope stability is here discussed in detail. The Nya Slussen (New water lock) project is located in the centre of Stockholm. An existing subway line is running through the construction area, and an excavation was carried out in the next to the metro embankment and foundation of the metro station superstructure. The slope of the excavation next to the subway was stabilized through soil nailing and shotcrete. Figure 2 shows one of the cross-sections of the excavation. The soil nailing was carried out in a gravel fill, but further beneath the slope are layers of organic clay and mud. Soil parameters were obtained and decided from CPT tests during pre-construction investigation and conservatively chosen characteristic values for design are shown in Table 1 . It is known that great uncertainties exist within soil parameters because of the heterogeneity of the organic clay and mud, which has been observed in similar soil stratifications, e.g. [18]. The layer of cohesive soil beneath the fill layer resulted in risk of a slip circle extending further down into the soil strata with resulting low stability of the slope. The case study concerns the inverse analysis of the stability of this cross-section.

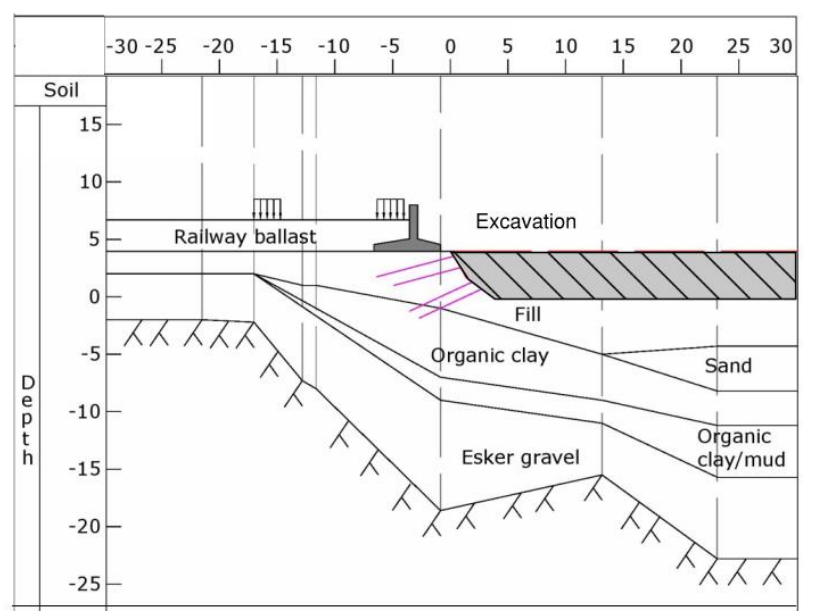

Fig. 2. A cross-section of the excavation next to the metro embankment and metro station foundation.

Table 1. Characteristic values of soil parameters in the slope stability calculations.

\begin{tabular}{|c|c|c|}
\hline Soil type & Parameter & Value \\
\hline \multirow{2}{*}{ Fill } & Friction angle & $32^{\circ}$ \\
\cline { 2 - 3 } Organic clay & Young's Modulus & $18 \mathrm{MPa}$ \\
\cline { 2 - 3 } & Undrained shear strength & $50 \mathrm{kPa}$ \\
\hline \multirow{2}{*}{$\begin{array}{c}\text { Organic } \\
\text { clay/mud }\end{array}$} & Young's Modulus & $18 \mathrm{MPa}$ \\
\cline { 2 - 3 } & Young's Modulus & $18 \mathrm{kPa}$ \\
\hline \multirow{2}{*}{ Sand } & Friction angle & $35.6^{\circ}$ \\
\cline { 2 - 3 } & Young's Modulus & $23.3 \mathrm{MPa}$ \\
\hline \multirow{2}{*}{ Esker gravel } & Friction angle & $33^{\circ}$ \\
\cline { 2 - 3 } & Young's Modulus & $81.5 \mathrm{MPa}$ \\
\hline
\end{tabular}

Design was carried out through slope stability calculations with both a standard slip-surface model and a finite element model in the program Plaxis 2D [19]. The slip surface model was executed with LRFD calculations according to the Eurocode 7, and the FEmodel with characteristic soil parameters using the 
strength reduction method outlined in [20]. This article is limited to the FE-model with characteristic input data in Table 1. The model calculations resulted in a total factor of safety of 1.67, which was deemed sufficient according to the national annex of the Eurocode. The incremental strain at collapse in shown in Figure 3.

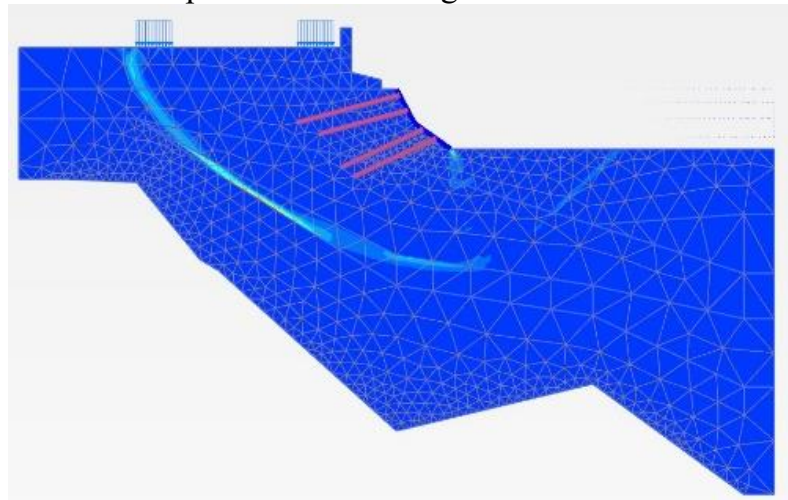

Fig. 3. Incremental strain of the slope at the onset of collapse.

Intensive monitoring was carried out at the slope due to its safety class and location next to the metro. Slope inclinometers ITS series HD were chosen to monitor the horizontal displacement the slopes. One of the inclinometers will be studied in the next section.

Significant uncertainties remained regarding the stability of the slope: The clay and mud layers where mixed and intertwined with the surrounding gravel and esker layers, hence the boundaries between different soil layers were not well defined. The boreholes itself displayed heterogeneity of the soil properties, and it was not possible to conduct Cone Penetration tests (CPTs) through all soil layers due to the large amounts of boulders in the fill layers. The high risk of the excavation location added to the uncertainties of the slope stability calculation necessitated close observation of the field measurements as well as some type of updated numerical model based on the behaviour of the response of the measurements. A simplified semiprobabilistic methodology for systematic assessment of the slope stability was therefore developed.

\section{$4 \quad$ Simplified methodology for the continuous assessment of slope stability}

In this chapter, a simplified semi-probabilistic methodology for slope stability assessment is elaborated, including the coupling between the measurements and the model calculations. The methodological framework is divided into three main parts: deterministic calculation, inverse calculation and forward calculation, following the link between the inverse and forward analysis. The probabilistic methods are used to decrease the uncertainties from deterministic design and estimate the model parameters with the field measurements as a reference. A new total factor of safety is finally calculated from forward analysis as the improved estimation of slope stability with prior information. Figure 4 shows the methodological framework in a flow chart.

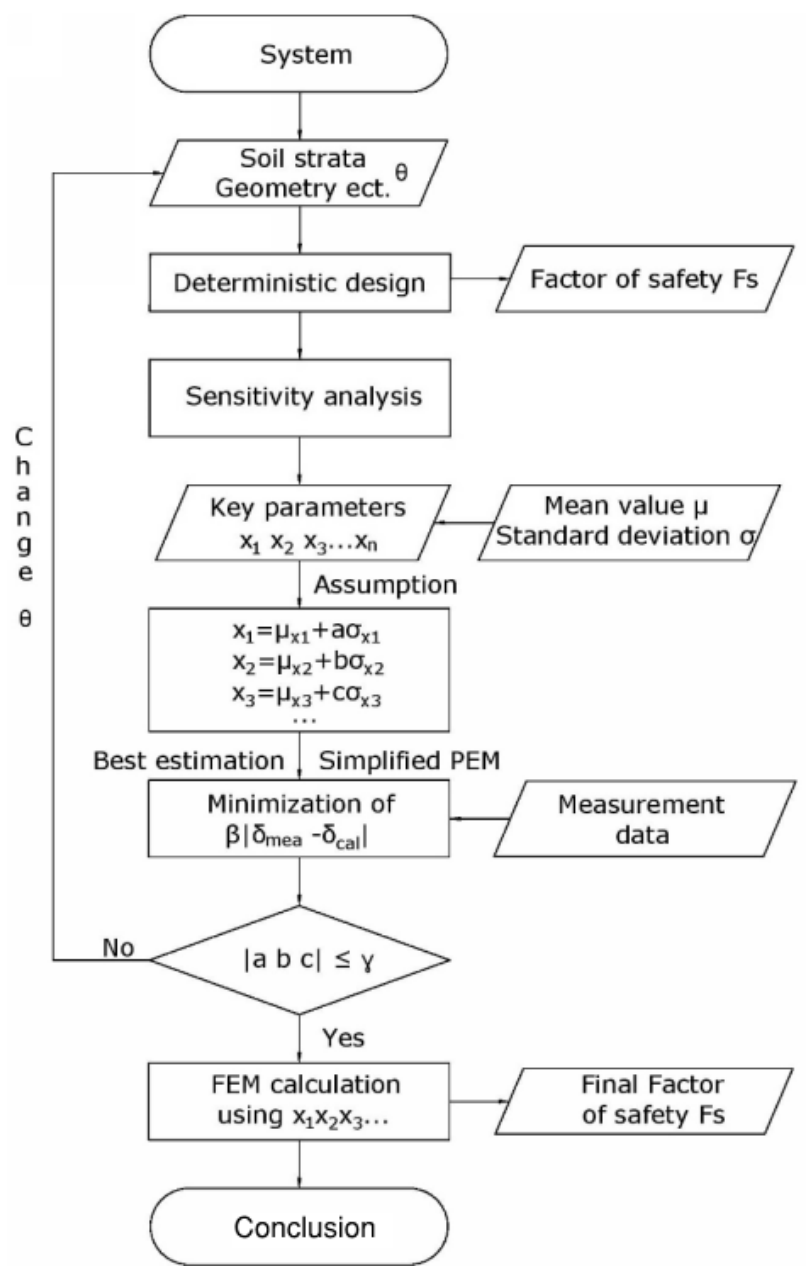

Fig. 4. A flowchart of the methodological framework.

The framework applies the program Plaxis 2D as the calculation tool to link the field measurements and the model prediction through the steps described here.

\subsection{Initial deterministic calculation}

This step was described in the previous section. The input data, including fixed parameters such as the geometry and the ground water table and the initial estimates of the soil parameters are obtained from preconstruction investigation. Some parameters (here named $\theta$ ) are fixed to reduce the degree of freedom of the system to idealise the highly non-linear system manageable throughout the methodological framework. A factor of safety (FS) for the slope is subsequently obtained from the FE-simulation with the strength reduction method, [20]. This is named the subjective calculation in the flowchart, since the resulting factor of safety depends on the subjective choice of characteristic soil parameters, hence belief in the model depends on the subjective judgement of the parameters.

\subsection{Inverse calculation}

The inverse calculation follows the approach exhibited in $[5,17]$, in which the accumulated vector norm between the observations (field measurements) and the 
model output is calculated for a set of estimated parameters $x_{i}$ and fixed parameters $\theta$ and where the best estimate of the parameters $x_{i}$ results from a minimization of the vector norm $D(\theta)$. The field measurement input data includes significant disturbances, and an adjusted method called the extended Bayesian method was used [17] according to equation 1.

$$
\min D(\theta)=\min \Sigma \beta_{i} \mid \delta_{\text {mea }}(t)-\delta_{\text {cal }}\left(x_{i}, t \mid \theta\right)
$$

where $\theta$ is the fixed model parameter such as model geometry and drainage conditions, $x_{i}$ are the input variables, while $\beta_{i}$ is the corresponding factor that adjusts the significance of the variables $x_{i}$. The factor $\beta_{i}$ is used to extend the normal Bayesian model in order to adjust from observations that result from measurement inaccuracies or actions which cannot be efficiently simulated. Weighting of the different components is introduced with a weight factor $\beta_{i}(0<\beta i<1) . \quad \delta_{\text {mea }}$ and $\delta_{\text {cal }}$ are for the measured and calculated deformations respectively, obtained at the $t$ with the estimate of the model parameters $x_{i}$.

A simplified parameter set inspired by Point Estimation Method (PEM) is then used to choose the different estimates of the model parameters $x_{i}$, [21]. The model parameters $x_{i}$ with mean values $\mu_{x i}$ and standard deviations $\sigma_{x i}$ are assumed to be known from preconstruction investigations. The purpose is to minimize Equation 1 over the distributions of the parameters $x_{i}$. Each parameter must be estimated at two points $\left(\mu_{x i}+\right.$ $\left.\sigma_{x i}\right)$ and $\left.\mu_{x i}-\sigma_{x i}\right)$ with $2^{n}$ combinations of $\mathrm{n}$ parameters. The weighting factor $\beta_{i}$ in Equation 1 is set at a lower value for observational values that appear incorrect.

After the point estimation, each parameter is assigned with a factor $(a, b, c \ldots)$, i.e. $x_{i}=\mu_{x i}+(a, b, c \ldots) \cdot \sigma_{x i}$. The best estimate of these factors $(a, b, c \ldots)$ is the obtained with respect to the minimization of the vector norm in Equation 1. If the absolute values of those factors are more than a limit value $\gamma$, the fixed parameters $\theta$ are probably incorrect and should be changed, according to the flowchart in Figure 4. Assume that normal distribution is hypothesized to all the parameters, there is only the $5 \%$ possibility that values will fall out of the interval of $\mu_{x i} \pm 2 \sigma_{x i}$. The fixed model parameters are then modified, and the simulations are iterated until an estimate that fulfils the requirement has been found according to Figure 4.

\subsection{Forward calculation}

Finally, the forward calculation is executed with the final estimated model parameters $x_{i}=\mu_{x i}+(a, b, c \ldots) \cdot \sigma_{x i}$ to obtained the final factor of safety $F_{S}$. The represents the updated factors of safety obtained through the best estimate of the model parameters $x_{i}$ and the fixed parameters $\theta$.

\section{Application of the methodology}

The methodological framework for systematic slope stability assessment will be applied to the slope shown in Figure 2 to verify its feasibility. The calculation of the initial factor of safety has already been elaborated. The inclinometer was installed near the slope to monitor its horizontal displacement. In order to visualize how deformation develops with construction phases, measuring data was taken following the excavation process and every month after final excavation. The inclinometer was installed on 2017-10-06 and had 14 levels between $3.915 \mathrm{~m}$ and $-9.085 \mathrm{~m}$ with $1 \mathrm{~m}$ interval. Horizontal displacement started developing within the excavation process within a few days after the installation after the excavation started and the slope was unreinforced before application of the shotcrete, shown in Figure 5.

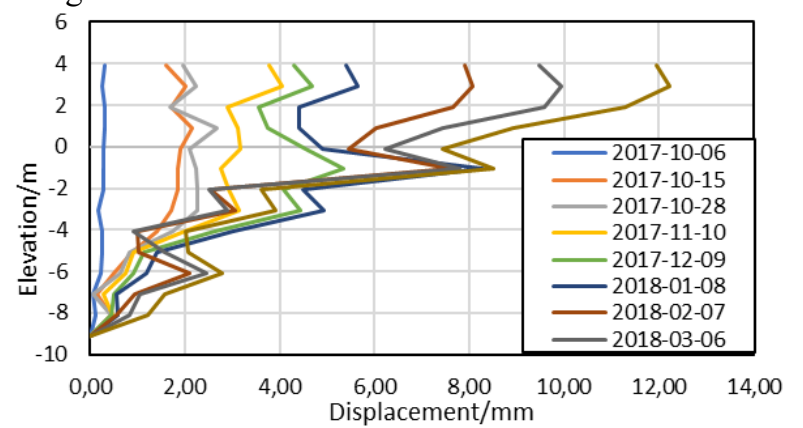

Fig. 5. Horizontal displacements of the inclinometer.

The soil parameters $c_{u}$, and $E$ of the organic clay and mud layer and the friction angle $\phi_{\text {fill }}$ of the fill layer were used as the model parameters $x_{i}$ in the model. The statistical properties of the model were obtained from CPT tests that performed before construction. These are shown in Table 2.

Table 2. Assumptions proposed for soil parameters.

\begin{tabular}{|c|c|c|}
\hline Parameter & Mean value $\mu$ & Standard deviation $\sigma$ \\
\hline$c_{u}$ & $176.4 \mathrm{kPa}$ & $80 \mathrm{kPa}$ \\
\hline$E$ & $18 \mathrm{MPa}$ & $5.4 \mathrm{MPa}$ \\
\hline$\varphi_{\text {fill }}$ & $34.4^{\circ}$ & $1.4^{\circ}$ \\
\hline
\end{tabular}

Model factors $a, b$ and $c$ were then assigned to each model parameter through their mean values $\mu$ and standard deviations $\sigma$.

$$
\begin{aligned}
& c_{u}=\mu_{c u}+a \cdot \sigma_{c u} \\
& E=\mu_{E}+b \cdot \sigma_{E} \\
& \varphi_{\text {fill }}=\mu_{\varphi f i l l}+c \cdot \sigma_{\varphi f i l l}
\end{aligned}
$$

The methodology includes finding proper values of $a$ $b$ and $c$ so that the difference in the accumulated vector norm between the modelled and the measured displacement can be minimized according to Equation 1 . A corresponding weight factor $\beta_{i}$ was given to every elevation of inclinometer measurement. Values of weight factors were chosen manually depending on the subjective quality of measurement and the influence of disturbance during excavation of the slope before it was reinforced with shotcrete, which resulted in large horizontal displacements at the crest of the slope. 8-point estimation is applied for three different variables to be estimated. Additionally, the centre point with only mean values from all the parameters was considered to 
reaching a better estimation so that it becomes a 9-point Point Estimation, shown in table 3.

Table 3. Point estimation parameter values.

\begin{tabular}{|c|c|c|c|c|c|c|c|}
\hline \multirow[b]{2}{*}{ Point } & \multirow[b]{2}{*}{$a$} & \multirow[b]{2}{*}{$b$} & \multirow[b]{2}{*}{$c$} & \multicolumn{2}{|c|}{$c_{u} / k P a$} & \multirow{2}{*}{$\begin{array}{c}E / \\
M P a\end{array}$} & \multirow{2}{*}{$\begin{array}{c}\varphi_{\text {fill }} \\
\circ\end{array}$} \\
\hline & & & & $\begin{array}{l}\text { Org } \\
\text { clay }\end{array}$ & $\begin{array}{l}\text { Clay/ } \\
\text { Mud }\end{array}$ & & \\
\hline 1 & 0 & 0 & 0 & 176.4 & 141.12 & 18 & 34.4 \\
\hline 2 & 1 & 1 & 1 & 256.4 & 205.12 & 23.4 & 35.8 \\
\hline 3 & -1 & 1 & 1 & 96.4 & 77.12 & 23.4 & 35.8 \\
\hline 4 & 1 & -1 & 1 & 256.4 & 205.12 & 12.6 & 35.8 \\
\hline 5 & 1 & 1 & -1 & 256.4 & 205.12 & 23.4 & 33 \\
\hline 6 & 1 & -1 & -1 & 256.4 & 205.12 & 12.6 & 33 \\
\hline 7 & -1 & 1 & -1 & 96.4 & 77.12 & 23.4 & 33 \\
\hline 8 & -1 & -1 & 1 & 96.4 & 77.12 & 12.6 & 35.8 \\
\hline 9 & -1 & -1 & -1 & 96.4 & 77.12 & 12.6 & 33 \\
\hline
\end{tabular}

The horizontal deformations at each time step and the factor of safety (through the strength reduction method [21]) were simulated in the FE-model. However, the model showed big differences between the measurement and the simulation near ground surface level. As a result, weight factors $\beta_{i}$ were applied to each elevation level with low values near surface, starting at $\beta_{i}=0.2$, and then increasing to $\beta_{i}=1$ at deeper soil levels.

The accumulated vector norm and the factor of safety for each estimation point according to Table 3 are shown in Figure 6. Point 7 in Table 3 displayed the lowest total difference between measurement and simulations (126.713 mm) along with a medium factor of safety (2.216).

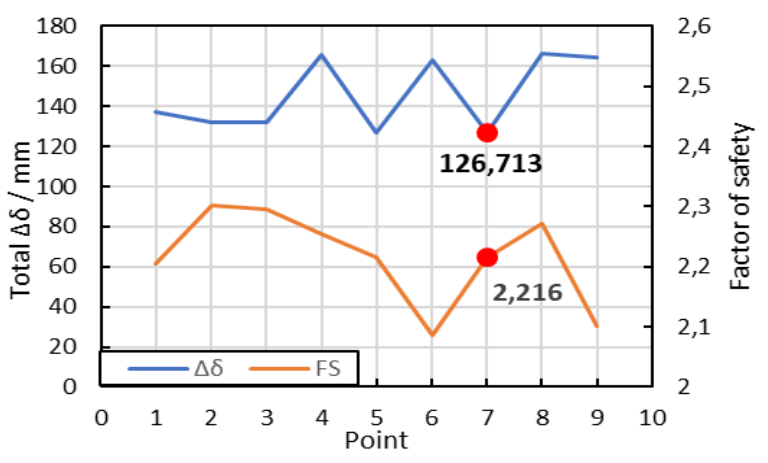

Fig. 6. The results from the 9-point estimate.

\section{Discussion}

The results in Figure 6 are similar to the results from a traditional sensitivity analysis, but also includes a criterion regarding the probability of each parameter set in the accumulated vector norm. The likelihood of each parameter set can therefore be studied. The minimum factors of safety are associated with higher accumulated vector norm, implying that they are less likely to occur, which is noticeable and helpful. The calculated final factor of safety is significantly higher than the factor of safety calculated with the conservatively chosen characteristic values (2.22 compared to 1.67$)$. The slope therefore appears to be safer in comparison to the initial calculation. The question is however to value the justified belief in the estimated values in the calculation according to [13-14]. The use of the probability criterion significantly increases the belief in the parameter set, since a larger distribution of parameter sets have been included.

The non-linear system resulting from the slope collapse has here been simplified to three main parameters and many factors that influence the stability of the slope have been set to fixed values $\theta$. This includes the stratification, the ground water table and the loads, and several other fixed model parameters could obviously influence the results of the slope stability model. This idealisation is however required in order to limit the calculations needed to study the whole parameter set.

\section{Conclusions}

A methodological framework for systematic slope stability assessments has been elaborated and implemented for a case study. The results show the feasibility of the methodological framework and has resulted in a transparent sensitivity study of the model parameters accompanied by a criterion showing the relative probability of the parameter estimated. The method is efficient and required only limited modelling and calculation time once the FE-model is created. However, a highly non-linear problem is idealized to a limited set of model parameters, and the choice of both model parameters and fixed parameters should be carried out with some scepticism.

The staff at Skanska Sverige AB are acknowledged for their kind held during the study of the slope.

\section{References}

1. Terzaghi, K., \& Peck, R. B. Soil mechanics in engineering practice. J. Wiley and Sons. (1948).

2. Ledesma, A., Gens, A., \& Alonso, E. E. Estimation of parameters in geotechnical backanalysis-I. Maximum likelihood approach. Computers and Geotechnics, 18(1), 1-27 (1996).

3. Gens, A., Ledesma, A., \& Alonso, E. E. Estimation of parameters in geotechnical backanalysis-II. Application to a tunnel excavation problem. Computers and Geotechnics, 18(1), 29-46. (1996).

4. Ledesma, A., Gens, A., \& Alonso, E. E. Parameter and variance estimation in geotechnical backanalysis using prior information. International Journal for Numerical and Analytical Methods in Geomechanics, 20 (2), 119-141. (1996).

5. Ledesma, A., Gens, A., \& Alonso, E. E. Identification of parameters of nonlinear geotechnical models. Computer Methods and Advances in Geomechanics, 5, 1005-1010. (1991). 
6. Siegel, R. A., Robertson, R. J., \& Anderson, D. G. Slope stability investigations at a landfill in southern California. In Geotechnics of Waste Fills-Theory and Practice. ASTM International. (1990).

7. Fell, R., Hungr, O., Leroueil, S., \& Riemer, W. Keynote lecture-geotechnical engineering of the stability of natural slopes, and cuts and fills in soil. In ISRM International Symposium. International Society for Rock Mechanics. (2000).

8. Ng, C. W. W., Zhan, L. T., Bao, C. G., Fredlund, D. G., \& Gong, B. W. Performance of an unsaturated expansive soil slope subjected to artificial rainfall infiltration. Geotechnique, 53(2), 143-157. (2003).

9. Eberhardt, E. Twenty-ninth Canadian Geotechnical Colloquium: The role of advanced numerical methods and geotechnical field measurements in understanding complex deep-seated rock slope failure mechanisms. Canadian Geotechnical Journal, 45(4), 484-510. (2008).

10. Bernander, S., Svensk, I., Holmberg, G., Bernander, J., \& Isacsson, K. Shear strength and deformation properties of clays in direct shear tests at high strain rates. In International Conference on Soil Mechanics and Foundation Engineering, Balkema Publishers. Vol. 2, 987-990. (1985).

11. Dounias, G. T., Potts, D. M., \& Vaughan, P. R. The shear strength of soils containing undulating shear zones-A numerical study. Canadian Geotechnical Journal, 25(3), 550-558. (1988).

12. Farrar, D. E., \& Glauber, R. R. Multicollinearity in regression analysis: the problem revisited. The Review of Economic and Statistics, 92-107. (1967).

13. Russell, B. Human knowledge: Its scope and limits. Routledge. (1948).

14. Ayer, A. J. The problem of knowledge. Harmondsworth: Penguin books. (1956).

15. Gettier, E. L. Is justified true belief knowledge? analysis, 23(6), 121-123. (1963).

16. Gordon A.. Fenton, \& Griffiths, V. D. Risk assessment in geotechnical engineering. John Wiley \& Sons. (2008).

17. Honjo, Y., Wen-Tsung, L., \& Guha, S. Inverse analysis of an embankment on soft clay by extended Bayesian method. International Journal for Numerical and Analytical Methods in Geomechanics, 18(10), 709-734. (1994).

18. Lundberg, A. B., \& Li, Y. Probabilistic characterization of a soft Scandinavian clay supporting a light quay structure. Geotechnical safety and risk V. IOS Press, Amsterdam, 170-175. (2015).

19. Brinkgreve, R. B. J., \& Vermeer, P. A. Plaxis manual, Plaxis B.V. (1998).

20. Griffiths, D. V., \& Lane, P. A. Slope stability analysis by finite elements. Geotechnique, 49(3), 387-403. (1999).
21. Rosenblueth, E. Point estimates for probability moments. Proceedings of the National Academy of Sciences, 72(10), .3812-3814. (1975). 